\title{
STRUCTURE AND EMISSION OF PARSEC-SCALE JETS IN QUASARS
}

\author{
MAX CAMENZIND \\ Landessternwarte Königstuhl, D-69117 Heidelberg, Germany
}

\begin{abstract}
The moving knots observed in VLBI of compact quasars are due to off-axis substructures in the parsec-scale jets which are dragged along by the underlying plasma flow. This is suggested by detailed MHD models for the structure of relativistic jets on the parsec-scale that are based on exact solutions of the nonlinear Grad-Schlüter-Shafranov equation in the asymptotic domain. These jet models are characterized by a current-carrying core and a current-free envelope. The corresponding core-radius $R_{c}=u_{p} R_{L}$ is related to the poloidal jet velocity $u_{p}$ and the light cylinder radius $R_{L}$ generated by the rotation of the magnetic surfaces. The Poynting flux, which has an inhomogeneous distribution in the jet, provides an off-axis energy reservoir that could be tapped and converted into particle acceleration by non-axisymmetric instabilities in the core-jet structure. Evidence is presented that quasi-periodic synchrotron emission (in the IR-optical) and inverse Compton emission (in X-and $\gamma$-rays) from freshly accelerated electrons are due to a lighthouse effect of the rotating knots.
\end{abstract}

\section{Introduction}

Rapid optical and radio activity has now been observed during outbursts of a number of radio-loud quasars and BL Lac objects. There is some evidence that gamma-emission, detected by EGRET at energies of a few hundred $\mathrm{MeV}$, occurs simultaneously with this optical activity. Two years ago, we proposed for the first time that this rapid variability could be explained in terms of emission from plasma blobs which move relativistically within the parsec-scale jets of these objects (Camenzind \& Krockenberger 1992a,b). Due to the non-vanishing angular momentum of the plasma in MHD jets, the rotation of the plasma blob leads to a lighthouse effect for the beamed emission.

In the meantime, this idea has been tested for the well-documented optical outburst of the quasar 3C 345 beginning in 1991 and ending in 1993 (Schramm et al. 1993). This model can explain the appearance of equidistant peaks in the emission and the simultaneous increase in the radio lightcurve. Both features, VLBI knots and emission activity appear therefore related to the underlying structure of the parsec-scale jets. VLBI maps taken at successive epochs also show quite convincingly a correlation between flux outbursts seen in the far-infrared - optical region of the spectrum and the birth of a new superluminal knot in the parsecscale jet. In this sense, detailed monitoring of compact radio sources provides very powerful tests for the structure of the parsec-scale jets of these sources.

\section{Formation of Relativistic Jets in Quasars}

In the following we discuss the structure of relativistic jets in quasars and BL Lac objects following from the relativistic magnetic jet model developed in the last

257

T. J.-L. Courvoisier and A. Blecha: Multi-Wavelength Continuum Emission of AGN, 257-260.

(C) 1994 IAU. Printed in the Netherlands. 
years (Camenzind 1986; 1987; 1990; 1992; 1993a; Appl \& Camenzind 1993a,b). This is the only model for extragalactic jets that can account for the observed relativistic motion and the tight collimation required by VLBI measurements on the milliarcsecond level. According to this picture, jets are nothing else than magnetically confined plasma flows supported by currents of the order of $10^{18}$ Ampères. These axisymmetric jet channels are the natural carriers for the knots seen in superluminal motion. Non-axisymmetric perturbations in the jet channel are dragged along by the underlying plasma and the induced rotation is responsible for the observed flaring activity.

Relativistic jets are formed under quite unique conditions (Camenzind, 1990; 1993a). Relativistic speeds cannot be attained in pure hydrodynamic acceleration, magnetic fields are a necessary ingredient for jet formation. Dynamo action in the inner accretion disk creates a rotating magnetosphere which is filled with disk plasma. Along open flux surfaces, the plasma is accelerated to relativistic speeds and collimated outside the light cylinder $R_{L}=c / \Omega_{K}\left(R_{\mathrm{F}}\right)$ for footpoint radii $R_{\mathrm{F}}$ near the inner last stable orbit at $r_{\mathrm{ms}}$ around a rapidly rotating Kerr black hole

$$
R_{L}=c / \Omega^{F} \simeq 10 G M_{H} / c^{2} \simeq 1.5 \times 10^{15} \mathrm{~cm} M_{H} / 10^{9} M_{\odot} .
$$

As in the Newtonian case, axisymmetric MHD flows can be described in terms of several invariants. The rotating field itself forms axisymmetric flux surfaces which are either collimated by the toroidal magnetic field or external pressures (Appl \& Camenzind 1993b). Since the host galaxies of quasars are expected to be giant ellipticals - as demonstrated by $3 \mathrm{C} 273$ - these galaxies dispose of large cores with core radii of a few hundred parsecs. This entire region can be filled up with molecular gas forming in this way a molecular torus (Camenzind 1993b). Steep pressure walls around the rotational axis will collimate the outflowing disk winds into jets with extremely small opening angles on the parsec-scale.

Disk plasma streams along these collimated magnetic surfaces in such a way that the total energy and total angular momentum are conserved. The toroidal magnetic field in the flow also follows from conservation laws. It turns out that the magnetization $\sigma_{D}$ provided by the central accretion disk around a rapidly rotating black hole is the only parameter that is essential for the question of the outflow speed (Camenzind 1993a). Relativistic speeds can only be obtained for $\sigma_{D}>1$ with outflow speeds $\gamma_{j} \leq \sigma_{D}$. When the magnetization of the disk is low, $\sigma_{D}<1$, only nonrelativistic speeds will result, $\beta_{j} \simeq \sigma_{D}^{1 / 3}$.

In the innermost part of a galaxy, these newly created plasma flows are then probably collimated by the external pressure of the molecular torus. Jets would otherwise expand outside the light cylinder until their self-collimation radius were achieved (Appl \& Camenzind 1993b). 


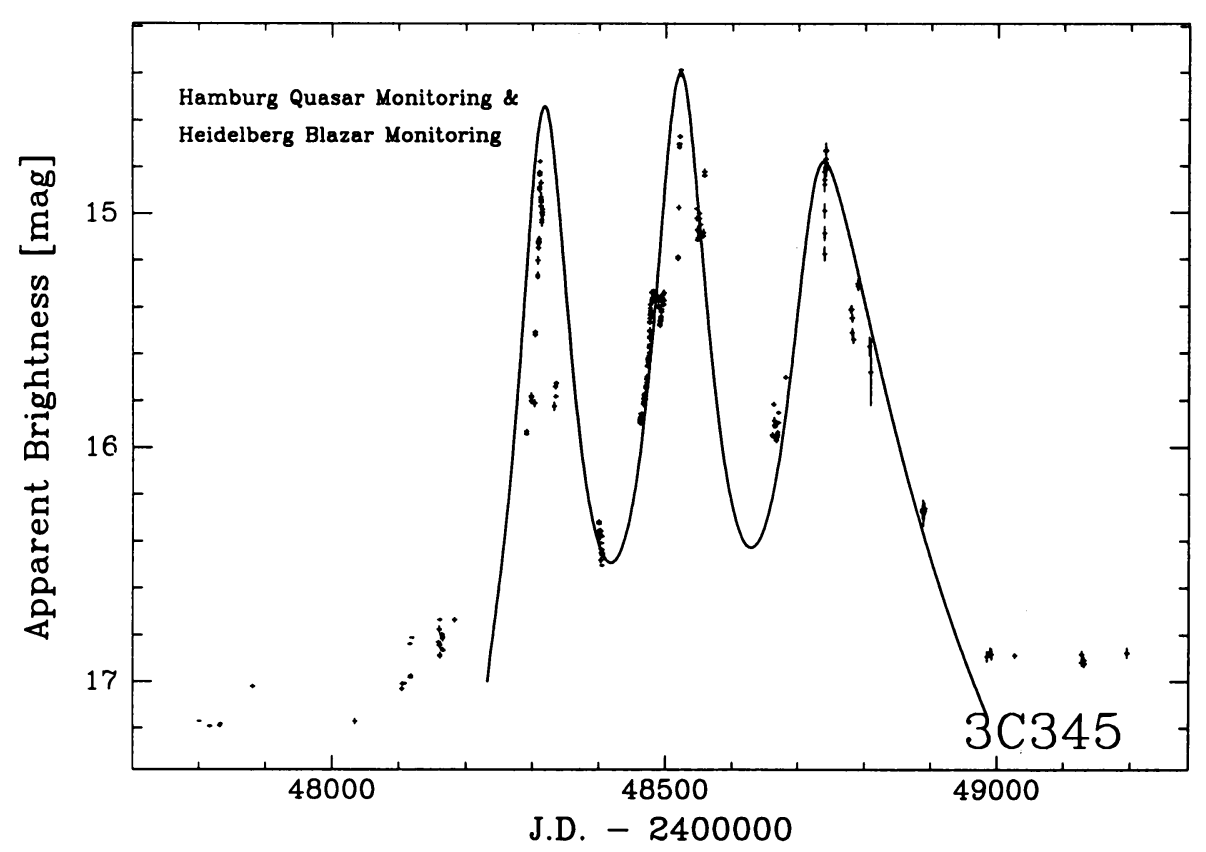

Fig. 1. A theoretical optical light curve (solid line) for $3 \mathrm{C} 345$ based on the lighthouse effect of a relativistic magnetized jet (Dreissigacker \& Camenzind 1994). The data points are from Schramm et al. (1993). A knot is moving with bulk Lorentz factor $\gamma_{j}=16$ at a radius $R_{0}=16 R_{L} \simeq 0.3$ pc for a central black hole $M_{H} \simeq 4 \times 10^{10} M_{\odot}$. The inclination angle between the line of sight and the jet axis is $0.95^{\circ}$. Equidistant peaks are produced by a completely collimated jet, which however opens up at a distance of $\simeq 50 \mathrm{pc}$ from the center resulting in a sudden decay of the optical synchrotron emission.

\section{Knot Propagation and Knot Emission}

The observed variability of radio-loud quasars in the non-thermal emission is interpreted in terms of substructures moving relativistically through the underlying jet channel. These structures need not be necessarily shocks, they could be created by instabilities (Appl \& Camenzind 1992) or plasmoids ejected along the magnetic surfaces. In the initial phase, the relativistic electrons in these structures are cooling by incoherent synchrotron emission and inverse Compton losses due to scattering of the electrons on infrared and UV-photons. Outbursts driven by such structures have been well documented for $3 \mathrm{C} 273,3 \mathrm{C} 345$ and many other sources. In the case of $3 \mathrm{C} 273$, the cycle time between various outbursts is of the order of two years, for 3C 345 somewhat longer. In the case of BL Lac objects, the cycle time is of the order of months or weeks.

The motion beyond the light cylinder occurs in such a way that the specific angular momentum stays constant. The rotation of such a bubble around the 
central axis of the jet produces therefore a lighthouse effect which mimics a timedependence of the received flux (Camenzind \& Krockenberger 1992a,b). The flux emitted by the bubble is quasi-periodic. The observed period appears drastically shortened due to the projection effect

$$
P_{\mathrm{obs}}=P_{\mathrm{jet}}(1+z)\left(1-\beta_{j} \cos \Theta\right) \simeq(1+z) \frac{P_{\mathrm{jet}}}{2 \gamma_{j}^{2}}
$$

This reduction in the time-scale is fundamental for the understanding of short time-scales observed in quasar emission. For knots moving with $\gamma_{j}=20$, all timescales appear shortened by a factor of $800-$

In Fig. 1 we show the optical light curve for 3C 345 (solid line) superposed onto the optical data for the last outburst starting in 1991 (Schramm et al. 1993). The first three peaks are practically equidistant in time requiring an initial intrinsic opening of the jet of $0.05^{\circ}$. The decay of the third peak and the absence of further peaks is due to a larger opening of the jet beyond a projected scale of 0.15 mas, corresponding to $\simeq 50$ parsecs away from the central source.

The detailed structure of the lightcurve (showing various precursors and shoulders) cannot be explained by this simple model. This time-structure in the lightcurve might signal the presence of a corresponding spatial substructure in these nonaxisymmetric knots. Such structures are expected to be formed, if the electrons accelerated to relativistic energies in these knots are due to magnetic reconnection. It is interesting that the amount of electrons needed to explain the observed synchrotron fluxes is of the order of the density found in the jet matter $\left(\simeq 10^{5} \mathrm{~cm}^{-3}\right)$. This would require that most of the available electrons are accelerated to relativistic energies in the knot. There are no pairs necessary to explain the synchrotron emission.

\section{References}

Appl, S., Camenzind, M.: 1992, Astron. Astrophys. 256, 354

Appl, S., Camenzind, M.: 1993a, Astron. Astrophys. 270, 71

Appl, S., Camenzind, M.: 1993b, Astron. Astrophys. 274, 699

Camenzind, M.: 1986, Astron. Astrophys. 162, 32

Camenzind, M.: 1987, Astron. Astrophys. 184, 341

Camenzind, M.: 1990, in G. Klare, ed., Rev. Mod. Astron. 3, 234 (Springer-Verlag, Heidelberg)

Camenzind, M.: 1992, in M. Locher, ed., Symp. Interface of Astrophys. with Nuclear and Particle Phys., p. 251 (PSI, Villigen)

Camenzind, M.: 1993a, in H.-J. Röser, K. Meisenheimer, eds., Lecture Notes in Physics 421,109

Camenzind, M.: 1993b, The Parsec-Scale Structure of Relativistic Jets in Quasars and the Origin of Gamma-Emission, preprint (Heidelberg)

Camenzind, M., Krockenberger, M.: 1992a, in W.J. Duschl, S.J. Wagner, eds., Physics of Active Galactic Nuclei, 551 (Springer-Verlag, Heidelberg)

Camenzind, M., Krockenberger, M.: 1992b, Astron. Astrophys. 255, 59

Dreissigacker, O., Camenzind, M.: 1994, in prep.

Schramm, K.-J., Borgeest, U., Camenzind, M., et al.: 1993, Astron. Astrophys. 278, 391 